\title{
Biobanks containing clinical specimens: defining characteristics, policies, and practices
}

\author{
Teresa Edwards, $\mathrm{MA}^{1}$, R. Jean Cadigan, $\mathrm{PhD}^{2}$, James $\mathrm{P}$ Evans, $\mathrm{MD}, \mathrm{PhD}^{3}$, and Gail \\ Henderson, $\mathrm{PhD}^{4}$
}

Teresa Edwards: teresa_edwards@unc.edu; R. Jean Cadigan: cadigan@ad.unc.edu; James P Evans: jpevans@med.unc.edu

${ }^{1}$ H.W. Odum Institute for Research in Social Science, University of North Carolina-Chapel Hill, C.B. 3355, Chapel Hill, NC 27599

${ }^{2}$ Department of Social Medicine, University of North Carolina-Chapel Hill, C.B. 7240, Chapel Hill, NC 27599

${ }^{3}$ Department of Genetics, University of North Carolina-Chapel Hill, C.B. 7264, Chapel Hill, NC 27599

\section{INTRODUCTION}

Biobanks - collections of human biological specimens stored for future research use - are crucial for biomedical advancement. Our prior research revealed that biobanks acquire specimens in a variety of ways [1]. One of the most common is to obtain residual or "leftover" specimens originally collected for clinical care from hospitals, clinical laboratories and pathology departments. However, there is a longstanding concern about the appropriateness of using stored specimens in ways not originally intended when they were collected [2-5].

In 1999, in response to remarkable developments in biomedical technologies applied to research with human specimens, the National Bioethics Advisory Commission issued a report on ethical and policy issues involving human biological materials [6]. It addressed the question of re-purposing clinical specimens for research use, and made three recommendations to assist interpretation of the Common Rule - the existing federal regulations governing human subjects protections. First, the Commission recommended that research conducted with unidentified clinical samples (that is, when identifying information is not collected or retained) not be deemed human subjects research and therefore not be regulated by the Common Rule. Second, the Commission recommended that research conducted with unlinked (or "anonymized") samples be defined as research on human subjects and regulated by the Common Rule, but eligible for exemption from Institutional Review Board (IRB) review. Finally, it recommended that research conducted with coded or identified samples be treated as research on human subjects and regulated by the Common Rule [6]. In 2004 (and later updated in 2008), these recommendations were reflected in guidance on secondary use of biospecimens issued by the federal Office for Human

(C) 2013 The Canadian Society of Clinical Chemists. Published by Elsevier Inc. All rights reserved.

${ }^{4}$ Corresponding Author. Department of Social Medicine, University of North Carolina-Chapel Hill, C.B. 7240, Chapel Hill, NC 27599. gail_henderson@med.unc.edu 919.843.8268.

Publisher's Disclaimer: This is a PDF file of an unedited manuscript that has been accepted for publication. As a service to our customers we are providing this early version of the manuscript. The manuscript will undergo copyediting, typesetting, and review of the resulting proof before it is published in its final citable form. Please note that during the production process errors may be discovered which could affect the content, and all legal disclaimers that apply to the journal pertain. 
Research Protections [7]. This guidance has important implications for whether and when consent must be obtained from contributors for the use of their samples.

Advances in genomics have continued to frame ethical considerations regarding informed consent and protection of contributor identities. Discoveries published in 2008 and later challenged the prevailing assumption that "de-identification" of samples containing genomic DNA is technically achievable, and therefore also challenged the adequacy of privacy protections that depend upon de-identification [8,9]. In July 2011, the federal government published an Advance Notice of Proposed Rule Making (ANPRM), entitled "Human Subjects Research Protections: Enhancing Protections for Research Subjects and Reducing Burden, Delay, and Ambiguity for Investigators," in the Federal Register [10,11]. Several of the proposed changes are highly relevant to the matters discussed here. One mandates written informed consent for any use of biospecimens, regardless of their identifiability and the original purpose for which they were obtained (i.e., clinical or research). Another proposes that consent should be obtained via a standardized form that would accommodate all future uses in a broad, open-ended manner. Response from the public was solicited, and over one thousand comments were submitted and posted. Many argued that mandated consent was "wholly unworkable," "... a logistical quagmire that will inhibit research," and that "The requirement for written permission to use de-identified specimens for research would profoundly impede clinical research for biomarkers and basic science studies." One lamented, "Loss of ability to use certain types of archived tissues without obtaining consent may be the death knell of live-saving translational research" (emphasis added). ${ }^{1}$

While controversy over how to adequately protect contributor identifiability continues, the environments in which such collections exist are also quite complex. Biospecimens acquired from clinical sources may be collected by pathology departments and clinical laboratories in academic medical centers, by biobanks associated with cancer centers or repositories focused on other types of patient populations, or by researchers with specific clinical research aims. Specimens may be stored with no explicit research intent, or acquired specifically for research purposes; collections may include specimens from a single source or multiple ones [1]. Biobanks' most salient feature is to store specimens for research purposes; they are challenged to ensure that research use of specimens from clinical sources adheres to federal guidelines. At the same time, clinical laboratories, whose most salient feature is to provide clinical diagnostic services, are increasingly challenged to consider how their clinical collections may be used by biobanks, and the extent to which consent for clinical uses anticipates such applications [12].

Little is known about biobanks that store specimens from clinical sources. There are no national data on these banks, nor even a unified definition. A number of authors have acknowledged the diversity of biobanks in the U.S. [13-16], which our 2012 national survey of U.S. biobanks demonstrated empirically $[1,17,18]$. In this paper, we address the particular ethical issues described above, focusing on biobanks in the 2012 survey that store collections from clinical sources-either solely or in conjunction with specimens from other sources.

\section{METHODS}

In 2012 we conducted a survey of U.S. biobanks--which we define as "organizations that acquire and store human specimens and associated data for future research use." We identified 636 eligible biobanks and 456 (72\%) responded to our survey. Details on biobank

\footnotetext{
${ }^{1}$ These quotes come from ANPRM comments that two of the authors analyzed for another project examining commenters' responses to the proposal to mandate written informed consent for the collection of all human specimens.
} 
identification and survey data collection may be found elsewhere [1]. In this paper we present simple response frequencies, with percentages where appropriate. Where percentages do not add to 100, it is due to rounding. Analyses were conducted in SAS version 9.2 (SAS Institute Inc., Cary, NC, USA).

\section{RESULTS}

\subsection{SPECIMEN COLLECTIONS}

In identifying biobanks for our study, we tried to eliminate organizations that serve solely as storage facilities for individual researchers - ones in which a researcher deposits specimens until they are needed, with no possibility that the specimens could be shared with others. We did not place any requirement on how the biobank acquires its specimens in order to be eligible for our study. Within the survey, we asked whether any of the specimens in the biobank's collection come from each of the following sources:

1. Hospitals, clinical laboratories, or pathology departments providing residual specimens from clinical care

2. Public health departments or programs providing residual specimens

3. Individuals providing specimens directly to the biobank

4. Any other source(s).

For this paper, we focus only on the 261 biobank managers who said "yes" to the first source. As shown in Table 1, forty-three respondents (16\%) reported that their biobank acquires specimens only from hospitals, clinical laboratories, or pathology departments. One hundred thirty-eight banks (53\%) acquire specimens from clinical settings and from individuals providing specimens directly. Thirty-nine (15\%) acquire them from clinical settings, from individuals directly, and from "other" sources; twenty-seven (10\%) acquire specimens from clinical and other sources. Only 7 biobanks (3\%) acquire specimens from all four sources, and the remaining 7 biobanks (3\%) acquire from clinical and public health settings, with or without collecting from individuals as well. Since we did not ask the respondent to indicate the number of specimens acquired from clinical settings relative to others in their collection, we do not know, for the 218 biobanks with mixed sources, whether the clinical specimens form only a small portion of their collection, nearly the entirety of it, or something in between.

Table 2 shows the number and percentage of biobanks storing various types of biological specimens. Solid tissue and serum/plasma are the most common. Twenty-four biobanks reported "other biological specimens," meaning they store something not listed on our survey. Nineteen of these wrote in a description of the "other" specimens, which are detailed in Table 3. Most biobanks (88\%) store more than one type of specimen. As shown in Table 4 , biobanks reported as many as 10 different types of specimens in their collections. The size of the specimen collection (number of specimens currently in storage) ranged from only 20 specimens to 54 million. Table 5 provides more detail on the number of specimens in storage. The range most frequently reported by the surveyed biobanks (19\%) was that of $10,000-49,999$ specimens. Due to a few very large banks, the mean number of specimens is 610,245 but the median is 7650 .

Also of interest in describing a biobank's collection is whether pediatric and/or post mortem specimens are included. In our survey, six biobanks (2\%) house exclusively pediatric specimens, and an additional $44 \%$ include some pediatric specimens. Seven percent of biobanks report collections composed entirely of specimens that were collected post mortem; an additional 37\% contain some post mortem specimens. Thus we found 
tremendous variation in the size and nature of specimen collections among these biobanks housing clinical specimens

\subsection{ORGANIZATIONAL FEATURES AND PRACTICES}

Among the 261 biobanks with collections that include clinical specimens, $15(6 \%)$ are forprofit organizations; the remainder are not. The vast majority (89\%) are part of some larger organization - most often an academic institution or a hospital/health care organization.

Since our survey was primarily designed to study the ethical, legal, and social issues surrounding biobanking rather than technical processes, we did not gather detailed information about the biobank's day-to-day operations. We did, however, ask a few questions which provide a snapshot of the general operations of these biobanks and the policies and practices in place. We asked for an approximation of the number of specimens the biobank received for processing in the past year. Responses ranged from zero to 5 million, with a mean of 42,486 and median of 500. Ninety-three percent of biobanks have standard operating procedures (SOPs) for processing their specimens. Eighty-four percent have a Laboratory Information Management System (LIMS), which we defined as "a computer-based inventory system that tracks the location and status of every specimen in the biobank." Seventy-five percent have standardized material transfer agreements (MTAs), and 80 percent have application forms through which researchers request specimens. Ninety-one percent of biobanks require studies that use their specimens to be approved by an Institutional Review Board (IRB). Only 33\%, however, have a formal business plan, and even fewer (27\%) have a written plan for what will happen to the specimens should the biobank be terminated for any reason. ${ }^{2}$

\subsection{INFORMED CONSENT}

Our survey included several items about whether and how informed consent is obtained for specimens stored by the biobank. Because procedures may not be the same for all specimens contained in a biobank's collection, we asked about typical consent procedures. We did not ask whether the biobank itself obtains consent from contributors, in recognition that a clinical staff member or other personnel might be the one to interact with the potential contributor rather than the biobank's own staff.

Figure 1 depicts our questions about informed consent, with corresponding response frequencies. Almost all biobanks (96\%) indicated that specimen contributors are typically informed that their specimens will be stored. Among those, an opt-in method (which we defined as "where contributors are asked for permission to store their specimens") is typically used (79\%), with 13\% using opt-out and 9\% reporting use of "both" or other methods unique to their situation. Among the biobanks using opt-in consent, most (79\%) use broad language which allows use for any future research purposes. Twelve percent use more limited language, $7 \%$ report use of both types, and 3\% wrote responses to the "other, specify" request, which were later renamed "tiered" because the contributor is typically given a choice about whether their specimens will be used only for a designated purpose or they may be used for other research aims that arise in the future.

\subsection{RETURN OF RESULTS}

For those biobanks that report having access to identifying information for any of its specimen contributors, we asked whether they return results to the individuals whose specimens are used for research studies. Only $15 \%$ of biobanks said "yes" when asked if

\footnotetext{
${ }^{2}$ An additional nineteen percent of biobank respondents were "not sure" whether a written plan exists.
} 
they "ever" return individual results to contributors. Thirty-four percent said they may (ever) return aggregate results.

Regardless of whether they report ever returning results, we asked biobanks whether they have a policy about returning individual results to contributors, and if so, what the policy states and whether it speaks to the issue of incidental findings, which we defined as "findings beyond the aims of the research." Figure 2 provides a graphic representation of the question sequence. Sixty-three percent of biobanks have policies about return of individual results to specimen contributors. In most cases (64\%), the policy states that such results will never be returned. In $33 \%$, results will be returned under certain conditions, and in only $3 \%$ will individual results always be returned.

In $48 \%$ of biobanks with policies about return of results, the policy addresses the question of incidental findings. Among policies of these 67 biobanks, 51\% state that incidental findings will never be returned, $46 \%$ specify it under certain conditions, and $3 \%$ state that incidental findings will always be returned.

\subsection{SPECIMEN AND TECHNOLOGY OWNERSHIP}

Who owns the specimens in a biobank? This question has garnered interest in the scientific community. We asked our survey respondents whether their biobank has a policy about specimen ownership. (See Figure 3.) For those who said yes, we asked who owns the specimens according to the policy; for those who said no, we asked the respondent's own opinion about ownership. We offered five response options and an "other, specify" category.

${ }^{3}$ Respondents could choose as many as they wished. Among those with written policies, the most commonly chosen response (52\%) was the larger organization that the biobank is a part of. Forty-three percent identified the biobank itself as an owner. Twenty-six percent identified the researcher who collected or deposited the specimens as an owner, and $8 \%$ chose the specimen contributor as an owner. For respondents who were asked their own opinion about ownership, the percentages vary slightly as shown in Figure 2. The differences could reflect discrepancy between policy and opinion, or could reflect the difference in organizations which have policies (potentially more formal ones) compared to those that do not.

We asked respondents a parallel question sequence about ownership of the rights to technologies developed from research using their biobank's specimens (See Figure 4). Among those with written policies, again, the most common response was the larger organization that the biobank is a part of $(69 \%)$ and next was the researcher or entity that developed the technology (44\%). The responses follow the same order but with slight differences in percentages among respondents who were asked their own views on technology ownership.

\subsection{USERS AND PERCEPTION OF UNDERUTILIZATION}

What level of specimen use is achieved by biobanks? We asked respondents how many requests for specimens they receive on average per year. The distribution of responses is shown in Table 6 . The most frequent response (26\%) was " 6 to 15 per year," followed by " 1 to 5 per year" (22\%) and "16 to 50 per year" $(20 \%)$. Thus, two thirds of these biobanks receive between 1 and 50 requests per year for their specimens. Most $(81 \%)$ report that researchers typically obtain specimens and data together; $17 \%$ report that requests are typically for specimens only, and only $2 \%$ of biobanks indicate that requests are typically for

\footnotetext{
${ }^{3}$ The response options for "larger organization biobank is part of" and "Network biobank belongs to" were only presented to biobanks which had previously indicated they were part of these.
} 
data only. Asked whether they typically approve (fulfill) all, some, or none of the requests they receive, $41 \%$ of biobanks report approving all requests, 58\% approve some, and 2 banks (1\%) approve none of the requests they receive. Forty-two percent of biobanks typically charge researchers for use of specimens (excluding shipping and handling); the remainder do not.

Who are the users of these biobanks containing clinical specimens? Thirty-six percent of the biobanks report that all requests they approve come from within their own organization or a larger organization of which they are a part. We asked the remaining 157 biobanks to identify the types of organizations from which approved requests came. As shown in Table 7, virtually all (96\%) biobanks had received one or more requests from academic researchers. Fifty-four percent had received request(s) from a researcher affiliated with a research institute; $48 \%$ had received requests from researchers in the federal government. Researchers in hospital/health care organizations or the pharmaceutical industry were also common (41\% and 40\%, respectively).

Based on qualitative work conducted prior to the survey [18], we posed the following question to biobank survey respondents:

We have heard from some biobanks that they are concerned that their specimen collection is being underutilized. How much of a concern is this for (biobank name)?

Fourteen percent of respondents felt that it was a major concern. The remainder were equally spread across categories of "a moderate concern," "a minor concern," or "not at all a concern." However, we do not know whether expressing little or no concern means the biobank receives the number of requests it deems appropriate, or that utilization rate is simply not of interest.

\subsection{DISPOSITION OF REMAINING SPECIMENS}

Biobanks differ in their policies regarding the disposition of remaining samples when the researcher has finished his or her research. Thirty-three percent of these biobanks require researchers to return remaining specimens. Eighteen percent require researchers to destroy the remaining samples, while the rest of the biobanks place no restrictions.

\section{DISCUSSION}

We present data from our 2012 U.S. biobank survey for the subset of biobanks that report storing specimens from clinical sources. Only $16 \%(\mathrm{~N}=43)$ indicated that this was their only source of specimens; over half reported that specimens were obtained from clinical sources and directly from individual specimen contributors. Other combinations were also reported, demonstrating the heterogeneity in composition of these biobank collections. Similar heterogeneity was documented regarding number and type of specimens stored, and number of requests for specimens per year. Only a few (6\%) are for-profit, and nearly $90 \%$ are part of larger organizations, mainly academic medical centers. Most report standardized systems for managing acquisition, storage, and release to researchers. Lastly, we document considerable heterogeneity in policies and practices regarding informed consent, return of research results, ownership of specimens and technology developed, utilization and disposition of remaining specimens.

Although unified federal regulations for human subjects protections, codified under the Common Rule, apply to biobank activities, acquisition of specimens and their release for research purposes is overseen by multiple institutional review boards (IRBs) who set policies in the context of both federal regulations and local needs. Variation in these policies 
is matched by dramatic differences in biobank goals, daily governance, and the diversity in composition of their specimen collections. Responding to this variation presents significant challenges for those who manage the collections and those who use biological samples for research.

Issues of consent are particularly challenging for those who maintain banked specimens. Commensurate with the significant heterogeneity observed in other aspects of biobanking, the type of consent by specimen contributors for research use varies considerably, although the majority of biobanks report using opt-in [1]. When a biobank is created to house research collections, contributors are often asked for their permission to store at the time of collection. In these cases, consent to storage for unspecified future research purposes is facilitated by broad or global permissions, and this appears to be the predominant type of consent overall [1], and for the sub-set of biobanks featured here. More controversy has surrounded opt-out approaches, defined as notification that specimens will be stored unless contributors refuse [19]. The controversy focuses on whether individuals truly understand that their specimens will be used for research, and reflects a longstanding concern about the appropriateness of using stored specimens in ways not originally intended when they were collected. These are more than just theoretical questions for those who direct biobanks as they must carefully balance facilitating research and protecting human subjects.

Another increasingly thorny issue for those who direct and use specimen repositories is whether they are responsible to return results to subjects when generated in the course of research studies [20]. A number of authors have pointed to the potential for this development to blur the boundary between clinical care and research, with uncertain implications for biobank policies and for clinical labs that contract with them. Such uncertain responsibilities are triggered when, as one author notes, "the diagnosis on a specimen during the course of review at a biobank differs from the original clinical diagnosis" [21]. In addition, while most such results transpire in research laboratories, CLIA (Clinical Laboratory Improvement Act) and other regulatory agencies mandate that results used for patient care be obtained in a certified laboratory by appropriate staff; some institutions have additional policies regarding this and restrict testing in research laboratories. Our survey results clearly document that existing policies at the level of individual biobanks are highly heterogeneous with regard to return of results. Such local variation may not contradict recommended principles and processes, yet broad community consensus is still desirable. Some recommendations have been put forward [13,22], but no firm, over-arching policies have yet been articulated. For the time-being, then, individual biobanks and their IRBs must formulate and document their own policies. Certainly those biobanks that do not retain identifying information about specimen contributors are not in the position to return results from research studies. Some biobanks may retain contact information, but choose not to return any results to subjects, while others develop policies for return of certain findings that when rising to the level of a clear, preventable harm, should be returned $[13,23]$. Whatever specific policies are adopted by an individual biobank, if that policy is to return research results to subjects, then the issue of CLIA confirmation must be addressed. The perceived advantages of research results to subjects must be balanced with practical realities such as a need for clinical personnel to evaluate and deliver such results to subjects - who then become patients - accentuating the blurring of research and clinical activities.

As we document, the world of this sub-set of 261 biobanks is highly variable in the types and numbers of specimens maintained and the policies that govern them. This uncertain and heterogeneous landscape demands careful consideration and planning by biobank managers to maintain high quality practices in acquisition, storage, and release of specimens all the while striving to protect the rights of subjects. Vaught and Lockhart, in a recent article, 
outline the importance of best practices in biobanking to improve research and clinical outcomes. While advocating for the application of NCI (National Cancer Institute) and ISBER (International Society for Biological and Environmental Repositories) standards for technical aspects, they also note, "In contrast to the more straightforward technical and management issues, ethical and regulatory practices often involve issues that are more controversial and difficult to standardize." [24] As we argue above, ethical and regulatory practices are developed within particular contexts, and are appropriately diverse. However, in order to evaluate this diversity, it is essential to understand practices currently being employed by biobanks that store clinical specimens.

\section{Acknowledgments}

We wish to thank the people who kindly participated in our survey, and members of our biobank research team at UNC, including Ian Conlon, Arlene Davis, Bryan Weiner, and Cathy Zimmer. Kriste Kuczynski created the figures and Warren Whipple assembled the references. Funding for this research was provided by several grants: 1R01HG005227-01A1 (G. Henderson, principal investigator, 'From Specimen to Biobank: Using An Organizational Perspective to Study ELSI Issues') from the National Human Genome Research Institute; 5UL1RR025747-04S1, a supplement to the UNC CTSA U54RR024382-01A1 (Runge, M., principal investigator, 'Enhancing Biobank Capacities Across CTSAs'); and the UNC Center for Genomics and Society, P50 HG004488, from the National Human Genome Research Institute. The content of this article does not necessarily reflect the views or policies of the funding agencies.

\section{References}

1. Henderson GE, Cadigan RJ, Edwards TP, Conlon I, Nelson AG, Evans JP, et al. Characterizing biobank organizations in the U.S.: results from a national survey. Genome Med. 2013; 5:3. [PubMed: 23351549]

2. Clayton E, Steinberg KK, Khoury MJ, Thomson E, Andrews L, Kahn MJE, et al. Informed consent for genetic research on stored tissue samples. J Am Med Assoc. 1995; 274:1786-1792.

3. American College of Medical Genetics, Storage of Genetics Materials Committee. ACMG statement. Statement on storage and use of genetic materials. American College of Medical Genetics Storage of Genetics Materials Committee. Am J Hum Genet. 1995; 57:1499-1500. [PubMed: 8533780]

4. Merz JF. Is Genetics Research “Minimal Risk”? IRB. 1996; 18:7-8. [PubMed: 11654745]

5. Merz JF, Sankar P, Yoo SS. Hospital Consent for Disclosure of Medical Records. J Law Med Ethics. 1998; 26:241-248. [PubMed: 11066882]

6. National Bioethics Advisory Commission. Research involving human biological materials: ethical issues and policy guidance. Rockv. MD: US Gov. Print. Off; 1999.

7. Office for Human Research Protections (OHRP). [Accessed Nov. 24, 2013] Guidance on Research Involving Coded Private Information or Biological Specimens. 2004. Available at http:// archive.hhs.gov/ohrp/humansubjects/guidance/cdebiol04.htm

8. Homer N, Szelinger S, Redman M, Duggan D, Tembe W, Muehling J, et al. Resolving Individuals Contributing Trace Amounts of DNA to Highly Complex Mixtures Using High-Density SNP Genotyping Microarrays. PLoS Genet. 2008; 4:e1000167. [PubMed: 18769715]

9. Gymrek M, McGuire AL, Golan D, Halperin E, Erlich Y. Identifying personal genomes by surname inference. Science. 2013; 339:321-324. [PubMed: 23329047]

10. Health and Human Services Department. Human Subjects Research Protections: Enhancing Protections for Research Subjects and Reducing Burden, Delay, and Ambiguity for Investigators. Fed Regist. 2011

11. Emanuel EJ, Menikoff J. Reforming the Regulations Governing Research with Human Subjects. N Engl J Med. 2011; 365:1145-1150. [PubMed: 21787202]

12. McDonald SA, Watson MA, Rossi J, Becker CM, Jaques DP, Pfeifer JD. A New Paradigm for Biospecimen Banking in the Personalized Medicine Era. Am J Clin Pathol. 2011; 136:679-684. [PubMed: 22031304] 
13. Wolf SM, Crock BN, Van Ness B, Lawrenz F, Kahn JP, Beskow LM, et al. Managing incidental findings and research results in genomic research involving biobanks and archived data sets. Genet Med. 2012; 14:361-384. [PubMed: 22436882]

14. Beskow LM, Burke W. Offering individual genetic research results: Context matters. Sci Transl Med. 2010; 2:38cm20.

15. Bledsoe MJ, Grizzle WE, Clark BJ, Zeps N. Practical implementation issues and challenges for biobanks in the return of individual research results. Genet Med. 2012; 14:478-483. [PubMed: 22323073]

16. Gibbons SMC. Regulating Biobanks: A Twelve-Point Typological Tool. Med Law Rev. 2009; 17:313-346. [PubMed: 19671622]

17. Boyer GJ, Whipple W, Cadigan RJ, Henderson GE. Biobanks in the United States: How to identify an undefined and rapidly evolving population. Biopreservation Biobanking. 2012; 10:511-517.

18. Cadigan RJ, Lassiter D, Haldeman K, Conlon I, Reavely E, Henderson GE. Neglected ethical issues in biobank management: Results from a U.S. study. Life Sci Soc Policy. 2013; 9:1-13.

19. Pulley J, Clayton E, Bernard GR, Roden DM, Masys DR. Principles of human subjects protections applied in an opt-out, de-identified biobank. Clin Transl Sci. 2010; 3:42-48. [PubMed: 20443953]

20. Wolf SM. The past, present, and future of the debate over return of research results and incidental findings. Genet Med. 2012; 14:355-357. [PubMed: 22481182]

21. Lockhart NC, Yassin R, Weil CJ, Compton CC. Intersection of biobanking and clinical care: should discrepant diagnoses and pathological findings be returned to research participants? Genet Med. 2012; 14:417-423. [PubMed: 22344228]

22. NCI Office of Biorepositories and Biospecimen Research. NCI best practices for biospecimen resources. 2011.

23. Evans JP, Rothschild BB. Return of results: not that complicated? Genet Med. 2012; 14:358-360. [PubMed: 22481183]

24. Vaught J, Lockhart NC. The evolution of biobanking best practices. Clin Chim Acta. 2012; 413:1569-1575. [PubMed: 22579478] 
- We present national survey data from 261 biobanks that store specimens from clinical sources.

- Most are part of larger academic organizations, and report standardized systems for managing acquisition, storage, and release to researchers.

- We found heterogeneity in the sources, number and type of specimens, and number of requests/year; and heterogeneity in policies and practices regarding informed consent, return of research results, ownership of specimens and technology developed, utilization, and disposition of remaining specimens.

- Responding to this variation presents significant challenges for those who manage collections. 


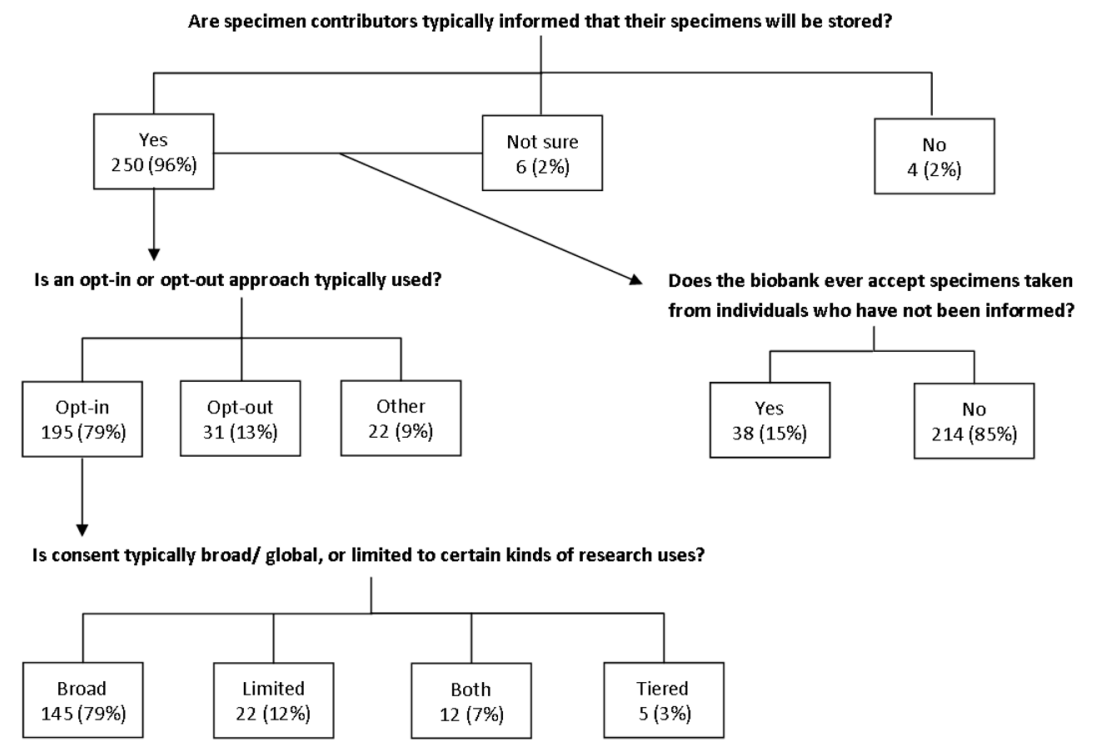

Figure 1.

Survey questions and response frequencies on contributor consent for specimen storage 


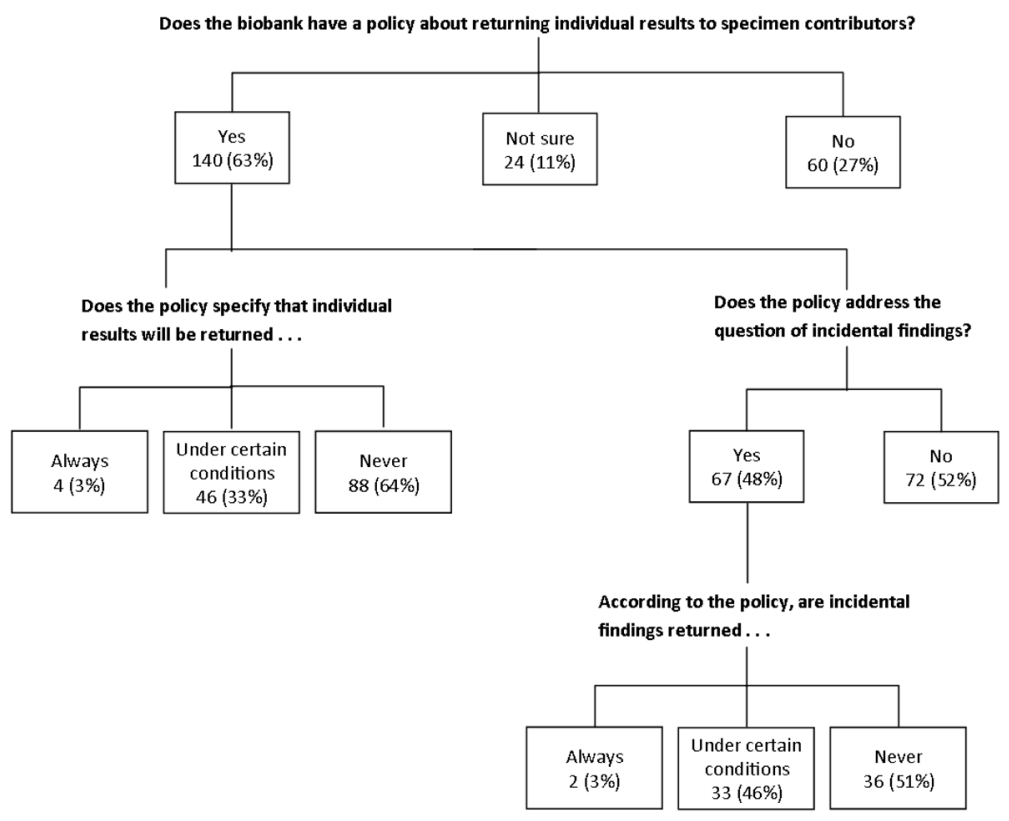

Figure 2.

Survey questions and response frequencies on return of individual results 




Figure 3.

Survey questions and response frequencies on specimen ownership 




Figure 4.

Survey questions and response frequencies on rights to new technologies 
Table 1

\section{Sources for acquisition of specimens}

\begin{tabular}{|l|r|r|}
\hline Source(s) & $\mathbf{n}$ & $\%$ \\
\hline Clinical and direct contributions & 138 & $53 \%$ \\
\hline Clinical only & 43 & $16 \%$ \\
\hline Clinical, direct contributions, and "other" & 39 & $15 \%$ \\
\hline Clinical and "other" & 27 & $10 \%$ \\
\hline Clinical, public health dept/program, direct contributions, and "other" & 7 & $3 \%$ \\
\hline Clinical and public health dept/program & 5 & $2 \%$ \\
\hline Clinical, public health dept/program, and "other" & 2 & $1 \%$ \\
\hline Total & 261 & $100 \%$ \\
\hline
\end{tabular}

Clinical: Hospitals, clinical laboratories, or pathology departments providing residual specimens from clinical care Public health dept/program: Public health departments or programs providing residual specimens

Direct contributions: Individuals providing specimens directly to the biobank

"Other": Any other source(s) 
Table 2

Types of specimens in storage (check all that apply)

\begin{tabular}{|l|r|r|}
\hline Percentage of biobanks storing specimens of this type & $\mathbf{n}$ & $\%$ \\
\hline Solid tissue specimens, including paraffin embedded, frozen, or other & 231 & $86 \%$ \\
\hline Serum or plasma & 203 & $79 \%$ \\
\hline Whole blood & 147 & $56 \%$ \\
\hline Peripheral blood cells or bone marrow & 138 & $53 \%$ \\
\hline Cell lines & 95 & $36 \%$ \\
\hline Saliva or buccal cells & 85 & $33 \%$ \\
\hline Urine or stool & 83 & $32 \%$ \\
\hline Cerebral spinal fluid & 59 & $23 \%$ \\
\hline Cord blood or cord blood derivatives & 30 & $11 \%$ \\
\hline Pathological body fluids & 27 & $10 \%$ \\
\hline Other biological specimens & 24 & $9 \%$ \\
\hline Hair/toenails & 6 & $2 \%$ \\
\hline
\end{tabular}


Table 3

Other types of biological specimens (Each type below was reported by only one biobank)

Amniotic fluid
Bronchoalveolar lavage
Monoclonal antibodies, biological research reagents
Muscle, hair, pituitary
Over 200 human, animal and environmental material types
Seminal plasma
Animal and human specimens of all kinds
Bacterial plasmids
Body fluids, tissue scrapings, aspirates, swabs, cultures, extracted DNA or RNA
Bone
Breast milk
Breast milk, meconium, dust, placenta, buffy coats, dried blood spot cards
Cell pellets
Cells cultured from biopsy samples collected from patients
Cervicovaginal lavage
Mucus
Prostatic fluid
Seminal vesicle, prostatic fluid, fresh tissue
Sperm, oocytes, embryos \& semen


Table 4

Number of types of specimens in storage

\begin{tabular}{|l|r|r|}
\hline & $\mathbf{n}$ & $\%$ \\
\hline 1 & 31 & $12 \%$ \\
\hline 2 & 32 & $12 \%$ \\
\hline 3 & 38 & $15 \%$ \\
\hline 4 & 45 & $17 \%$ \\
\hline 5 & 35 & $13 \%$ \\
\hline 6 & 34 & $13 \%$ \\
\hline 7 & 17 & $7 \%$ \\
\hline 8 & 12 & $5 \%$ \\
\hline 9 & 12 & $5 \%$ \\
\hline 10 & 3 & $1 \%$ \\
\hline Total & 260 & $100 \%$ \\
\hline
\end{tabular}


Table 5

Number of specimens in storage

\begin{tabular}{|l|r|r|}
\hline & $\mathbf{n}$ & $\boldsymbol{\%}$ \\
\hline Less than 500 & 30 & $12 \%$ \\
\hline $500-999$ & 19 & $8 \%$ \\
\hline $1000-1999$ & 23 & $9 \%$ \\
\hline $2000-4999$ & 34 & $14 \%$ \\
\hline $5000-9999$ & 24 & $10 \%$ \\
\hline $10,000-49,999$ & 48 & $19 \%$ \\
\hline $50,000-99,999$ & 24 & $10 \%$ \\
\hline $100,000-499,999$ & 30 & $12 \%$ \\
\hline $500,000+$ & 17 & $7 \%$ \\
\hline Total & 260 & $100 \%$ \\
\hline
\end{tabular}

Mean: 610,245

Median: 7650

Std deviation: 4,280,855

Range: 20 - 54,000,000 
Table 6

Number of Requests for Specimens and/or Data Received Per Year

\begin{tabular}{|l|r|r|}
\hline & $\mathbf{n}$ & $\%$ \\
\hline 0 per year & 6 & $2 \%$ \\
\hline 1 to 5 per year & 56 & $22 \%$ \\
\hline 6 to 15 per year & 65 & $26 \%$ \\
\hline 16 to 50 per year & 49 & $20 \%$ \\
\hline 51 to 100 per year & 32 & $13 \%$ \\
\hline 101 to 500 per year & 28 & $11 \%$ \\
\hline 501 to 1000 per year & 6 & $2 \%$ \\
\hline More than 1000 per year & 9 & $4 \%$ \\
\hline Total & 251 & $100 \%$ \\
\hline
\end{tabular}


Table 7

In the past year, have any of the approved requests come from researchers who work for...

\begin{tabular}{|l|r|r|}
\hline & $\mathbf{n}$ & $\boldsymbol{\%}$ \\
\hline Academic institutions (including medical centers and academic research centers) & 151 & $96 \%$ \\
\hline Research institutes (including contract research organizations) & 85 & $54 \%$ \\
\hline The federal government (including NIH and CDC) & 76 & $48 \%$ \\
\hline Hospitals or health care organizations & 65 & $41 \%$ \\
\hline Pharmaceutical companies & 63 & $40 \%$ \\
\hline Disease or health advocacy organizations & 18 & $11 \%$ \\
\hline State government (including public health departments) & 7 & $4 \%$ \\
\hline Insurance companies & 1 & $1 \%$ \\
\hline
\end{tabular}

Based on 157 biobanks who received this survey question. 